\title{
Two-step interferometry by a regularized optical flow algorithm
}

\author{
J. Vargas,$^{1, *}$ J. Antonio Quiroga, ${ }^{2}$ C. O. S. Sorzano, ${ }^{1}$ J. C. Estrada, ${ }^{3}$ and J. M. Carazo ${ }^{1}$ \\ ${ }^{1}$ Biocomputing Unit, Centro Nacional de Biotecnología-CSIC, C/ Darwin 3, 28049, Cantoblanco (Madrid), Spain \\ ${ }^{2}$ Optics Department, Universidad Complutense de Madrid, Facultad de CC. Físicas, Ciudad Universitaria s/n, 28040 Madrid, Spain \\ ${ }^{3}$ Centro de Investigaciones en Óptica A. C., Loma del Bosque 115, Col. Lomas del Campestre, 37150, León (Guanajuato), Mexico \\ *Corresponding author: jvargas@cnb.csic.es
}

Received July 5, 2011; accepted August 3, 2011;

posted August 9, 2011 (Doc. ID 150394); published September 1, 2011

\begin{abstract}
A two-step phase-shifting method, that can demodulate open- and closed-fringed patterns without local sign ambiguity is presented. The proposed method only requires a constant phase-shift between the two interferograms. This phase-shift does not need to be known and can take any value inside the range $(0,2 \pi)$, excluding the singular case where it corresponds to $\pi$. The proposed method is based on determining first the fringe direction map by a regularized optical flow algorithm. After that, we apply the spiral phase transform (SPT) to one of the fringe patterns and we determine its quadrature signal using the previously determined direction. The proposed technique has been applied to simulated and experimental interferograms obtaining satisfactory results. A complete MATLAB software package is provided in [http://goo.gl/Snnz7]. () 2011 Optical Society of America

OCIS codes: $100.5070,100.2650$.
\end{abstract}

Phase-shifting interferometry (PSI) is a useful technique in optical metrology for measuring the modulating phase of interferograms [1]. Typically, in standard PSI, we obtain a sequence of $\bar{N}$ interferograms with known and constant temporal carrier. A minimum of three phase-shifted interferograms are need to retrieve the phase. Other wellestablished phase-shifted methods are the 4-, 5-steps, for example. A good general overview of the phase-shifting algorithms can be seen in [2]. As a general rule, the use of few interferograms simplifies the computation process and reduces the processing speed in PSI. Moreover, it increases the robustness against uncontrolled mechanical vibrations, air turbulence, or temperature changes that are typical problems of interferometric setups. In the case of high-frequency open-fringe interferograms, there are well-known solutions as the Fourier transform method [3] but in the case of closed-fringes, we need at least-two frames to solve the local sign ambiguity if we do not have additional a priori information.

In the past, there have been reported works about

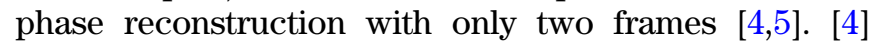
presents the standard technique for obtaining the phase map from two phase-shifted interferograms. This method is based on applying the Fourier transform demodulation approach to both interferograms. After that, the phasestep value is calculated for every pixel using a direct algebraic expression. Because the phase-step has to be constant along the full image, it is possible to solve the local sign ambiguity and then retrieve the phase map. In [4], the authors recommend using the method when the phase-step is inside the range $[\pi / 3,2 \pi / 3](\mathrm{rad})$. Additionally, this method is very sensitive to noise. In [5], a twostep method has been recently presented. This method consists of a self-tuning (ST) approach that first retrieves the phase-step between interferograms looking for the minimum of a merit function. Then a quadrature filter is constructed and the phase is determined. This method produces good results when the phase-step is close to $\pi / 2$ (rad), but the accuracy decreases when the phasestep moves away from this value. Additionally, the method requires that the interferograms were previously normalized.

In this Letter, we propose a two-step phase-shifting method that is capable of retrieving the modulating phase in the whole $(0,2 \pi)$ range in a robust and fast way. Our method, as any two-step method, cannot retrieve the phase when the phase-shift is exactly $\pi$ (rad). In this singular case there is not enough information. The method first obtains the fringe direction using a regularized optical flow method. After that, the sign ambiguity problem is solved and the modulating phase can be obtained from one interferogram using the spiral phase transform (SPT).

The optical flow approach is a standard method used in computer vision for obtaining the distribution of apparent velocities of objects, surfaces, and edges in an image set [6]. The optical flow method calculates the motion field at every pixel position between two image frames that are taken at times $t$ and $t+\Delta t$. An arbitrary pixel at time $t$ given by $(x, y, t)$ and with intensity $I(x, y, t)$ will move between two consecutive frames to the position $(x+\Delta x, y+\Delta y, t+\Delta t)$ with intensity $I(x+\Delta x, y+$ $\Delta y, t+\Delta t)$. Assuming the movement is small, we can expand the intensity map at $t+\Delta t$ as

$$
\begin{aligned}
I(x+\Delta x, y+\Delta y, t+\Delta t) \cong & I(x, y, t)+I_{x} \Delta x \\
& +I_{y} \Delta y+I_{t} \Delta t,
\end{aligned}
$$

where $I_{x}, I_{y}$, and $I_{t}$ represent the derivatives of $I$ with respect to $x, y$, and $t$. We suppose that the brightness of an object does not change along time; therefore, we have $I(x+\Delta x, y+\Delta y, t+\Delta t)=I(x, y, t)$ and Eq. (1) can be rewritten as

$$
I_{x} u+I_{y} v+I_{t}=0,
$$

where $u=x_{t}$ and $v=y_{t}$ corresponds to the velocity components. Equation (2) can be used to determine the components of the movement in the direction of the 
intensity gradient, but we cannot, however, obtain these components in the direction of the isointensity contours. As a consequence, the flow velocity $(u, v)$ cannot be computed locally without introducing additional constraints [6]. One possibility is to impose smoothness in the $u$ and $v$ fields so we can develop a regularized optical flow method. Suppose that we define an energy map as

$$
E^{2}=\left(I_{x} u+I_{y} v+I_{t}\right)^{2}+\lambda\left(u_{x}^{2}+u_{y}^{2}+v_{x}^{2}+v_{y}^{2}\right),
$$

where $E$ is the energy to be minimized and $\lambda$ is the regularizing parameter that weighs the smoothness of $u$ and $v$. From Eq. (3), it is possible to obtain an iterative solution using the Gauss-Seidel method [] $]$ as

$$
\begin{aligned}
u^{k+1} & =\bar{u}^{k}-I_{x}\left[I_{x} \bar{u}^{k}+I_{y} \bar{v}^{k}+I_{t}\right] /\left(\lambda^{2}+I_{x}^{2}+I_{y}^{2}\right), \\
v^{k+1} & =\bar{v}^{k}-I_{y}\left[I_{x} \bar{u}^{k}+I_{y} \bar{v}^{k}+I_{t}\right] /\left(\lambda^{2}+I_{x}^{2}+I_{y}^{2}\right),
\end{aligned}
$$

where $u^{k+1}$ and $v^{k+1}$ are the velocity components obtained in the iteration $k+1$ and $\bar{u}$ and $\bar{v}$ corresponds to the mean value of $u$ and $v$ in a defined neighborhood, typically of size of $5 \times 5 \mathrm{px}$. Once we have obtained $u$ and $v$ maps at every pixel we can obtain the direction map as

$$
\eta=\arctan \left(\frac{v}{u}\right)
$$

In two-step PSI we have two patterns of the form

$$
I_{t}=a+b \cos \left(\Phi+\omega_{0} t\right), \quad t=0,1,
$$

where $a$ is the background illumination, $b$ is the modulation term, $\Phi$ is the modulating phase, and $\omega_{0}$ is the instantaneous temporal frequency.

[7] introduced the SPT, which permits to transform a cosine signal into its quasi-quadrature signal. If we suppress the DC component of $I_{t}$ in Eq. (6) by using a high pass filter and apply the SPT operator, we obtain

$$
\operatorname{SPT}\left\{\tilde{I}_{t}\right\}=i \exp (i \eta) b \sin \left(\Phi+\omega_{0} t\right), \quad t=0,1,
$$

with $\tilde{I}_{t}=b \cos \left(\Phi+\omega_{o} t\right)$ and $\operatorname{SPT}\{\cdot\}$ the SPT that corresponds to

$$
\operatorname{SPT}\{\cdot\}=\mathrm{FT}^{-1}\left\{\left(\frac{\omega_{x}+i \omega_{y}}{\sqrt{\omega_{x}^{2}+\omega_{y}^{2}}}\right) \operatorname{FT}\{\cdot\}\right\},
$$

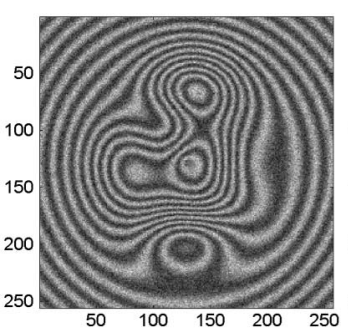

(a)

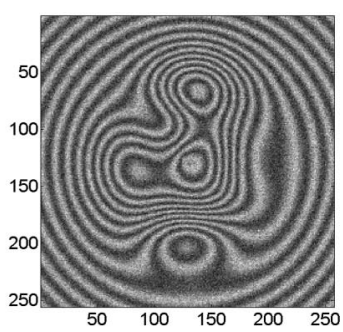

(b)
Fig. 1. Two fringe patterns used in the first simulation.

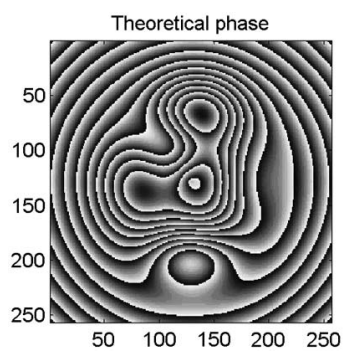

Fig. 2. Theoretical phase map of the simulated fringe patterns.

where $\eta$ is the direction map, FT means 2D Fourier transform, $\omega_{x}$, and $\omega_{y}$ are the coordinates in the Fourier space. Note that, if only there are one or two fringes in the interferograms we can always subtract the DC component normalizing the interferograms []ㅛ. In our case, we propose to calculate the direction map from two interferograms using the regularized optical flow method outlined above. From Eq. (7), if we know the direction map, we have

$$
b \sin \left(\Phi+\omega_{0} t\right)=-i \exp (-i \eta) \operatorname{SPT}\left\{\tilde{I}_{t}\right\} t=0,1,
$$

from which, we can obtain the modulating wrapped phase as

$$
\Phi=\arctan \left(\frac{-i \exp (-i \eta) \operatorname{SPT}\left\{\tilde{I}_{t}\right\}}{\tilde{I}_{t}}\right) t=0,1
$$

We have compared the results obtained by the proposed optical flow (OF) method with the results obtained by the standard Kreis [4] and the ST [5] methods. Figure 1 shows two sample fringe patterns with a phase-shift between them of $0.5 \mathrm{rad}$. The images' size are $256 \times 255$ pixels. The noise is Gaussian and additive and the signal-to-noise ratio (SNR) is $20 \%$.

Figure 2 shows the reference theoretical phase map and Fig. 3 shows the obtained phases by the OF (a), the Kreis (b), and the ST methods (c). Note that in Fig. 3 the wrapped phase retrieved by the Kreis and by the ST method are highly affected by noise.

The $r m s$ (root-mean-square) error of the difference between the theoretical and obtained wrapped phases are $0.53 \mathrm{rad}, 0.66 \mathrm{rad}$ and $0.84 \mathrm{rad}$ for the phases computed by the OF, Kreis, and ST methods, respectively. In this example, the processing times are respectively $0.53 \mathrm{~s}$, $0.20 \mathrm{~s}$, and $0.72 \mathrm{~s}$ using a $2.67 \mathrm{GHz}$ laptop and MATLAB.

In order to compare the robustness of the proposed method to different phase-shifting values between the fringe patterns, we have obtained the rms errors and

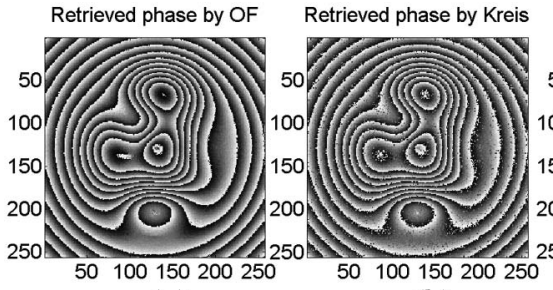

(a) (b)

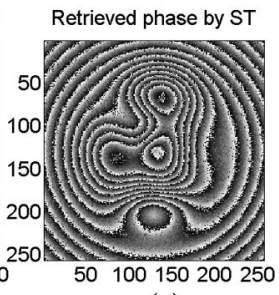

(c)
Fig. 3. Reconstructed wrapped phases by the proposed OF (a) by the Kreis, (b) by the self-tuning, and (c) methods. 


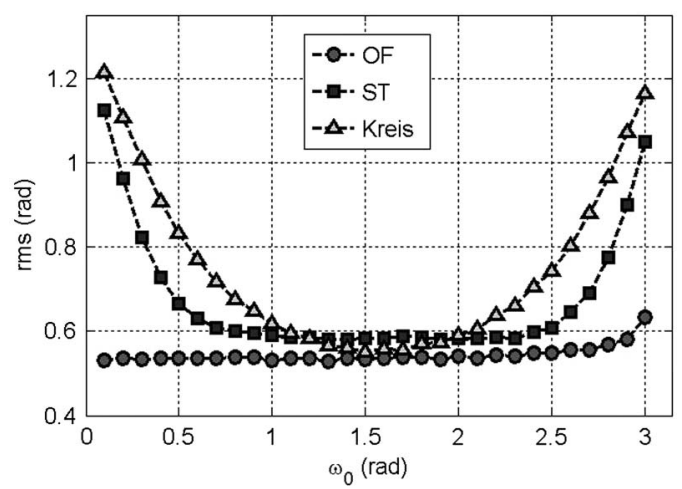

Fig. 4. Obtained $r m s$ errors by the proposed OF, Kreis, and self-tuning method for different temporal frequencies.

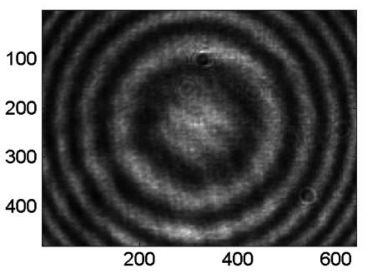

(a)

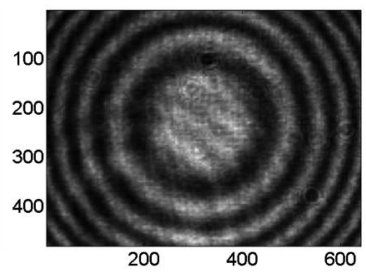

(b)
Fig. 5. Two real phase-shifted interferograms.

processing times for different phase-shifting values. The fringe patterns to process are similar to the ones shown in Fig. 1 in terms of SNR and theoretical phase map. Figure $\overline{4}$ shows the obtained $r m s$ errors for different temporal frequencies. As can be seen from Fig. 4, the obtained $r m s$ are always lower with the OF method than when using the Kreis or ST methods. Additionally, from Fig. 4, we see that the $r m s$ obtained by the OF method is practically constant along the full range.

We have applied the proposed algorithm to real interferograms. We have compared the retrieved phase from the proposed OF and Kreis two-step methods with the phase obtained by the AIA method [9] using nine phaseshifted interferograms. We denote the phase obtained by the AIA method as the reference phase. Figure 5 shows the used interferograms. In Figs. 6(a)-6(c), we show the obtained wrapped phases by the $\overline{\mathrm{OF}}$, the Kreis, and the least-squares method (reference phase). From Fig. $\underline{6}$, we can see a good agreement between the $\mathrm{OF}$ phase (a) and the reference phase (c). The rms error

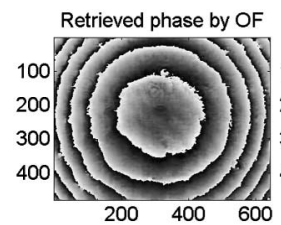

(a)

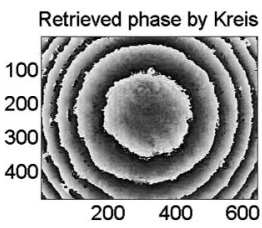

(b)

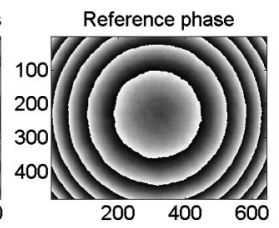

(c)
Fig. 6. Reconstructed wrapped phases by the proposed OF (a) by the Kreis and (b) by the least-squares method using nine interferograms (reference phase).

of the difference between the reference and the $\mathrm{OF}$ phases is $0.81 \mathrm{rad}$ and the processing time is $1.1 \mathrm{~s}$. On the other hand, the rms error obtained by the Kreis method is $0.84 \mathrm{rad}$ and the processing time is $0.65 \mathrm{~s}$.

In conclusion, we have proposed a two-step phaseshifting method based on a regularized optical flow algorithm that does not need to know the phase-shift between interferograms. Additionally, the retrieved phase error is practically independent of the temporal frequency or phase-step. We have tested the proposed method with simulated and real interferograms for different values of the phase-step. We have compared our algorithm with the Kreis [3] and ST [4] methods and we have shown a very good performance of the proposed method. All the examples of this work can be reproduced running the MATLAB package that can be found in [10].

We would like to acknowledge partial support from the Spanish Ministry of Science and Innovation through grants ACI2009-1022, ACI2010-108 and BIO2010-16566.

\section{References}

1. D. Malacara and M. Servín, and Z. Malacara, Interferogram Analysis for Optical Testing (Marcel Dekker, Inc, 1998).

2. M. Servin, J. C. Estrada, and J. A. Quiroga, Opt. Express 17, 21867 (2009).

3. M. Takeda, H. Ina, and S. Kobayashi, J. Opt. Soc. Am. 72 156 (1982).

4. T. M. Kreis and W. P. O. Jueptner, Proc. SPIE 1553, 263 (1992).

5. J. Vargas, J. A. Quiroga, T. Belenguer, M. Servín, and J. C. Estrada, Opt. Express 19, 638 (2011).

6. B. K. P. Horn and B. G. Schunck, Artif. Intell. 17, 185 (1981).

7. K. G. Larkin, D. J. Bone, and M. A. Oldfield, J. Opt. Soc. Am. A 18, 1862 (2001).

8. J. A. Quiroga and M. Servín, Opt. Commun. 224, 221 (2003).

9. Z. Y. Wang and B. T. Han, Opt. Lett. 29, 1671 (2004).

10. http://goo.gl/Snnz7. 